\section{Vibrio vulnificus necrotizing fasciitis associated with acupuncture}

\author{
Yael Kotton, ,1,2 Soboh Soboh, ${ }^{3}$ \\ Naiel Bisharat ${ }^{1,2}$ \\ 'Department of Medicine D, Emek \\ Medical Center, Afula; ${ }^{2}$ The Rappaport \\ Faculty of Medicine, Technion - Israel \\ Institute of Technology, Haifa; \\ ${ }^{3}$ Department of Medicine B, Baruch \\ Padeh Medical Center, Poriya, Tiberias, \\ Israel
}

\section{Abstract}

Necrotizing fasciitis is a severe life-threatening infection of the deep subcutaneous tissues and fascia. Infection with Vibrio vulnificus, a halophilic Gram-negative bacillus found worldwide in warm coastal waters, can lead to severe complications, particularly among patients with chronic liver diseases. We herein present an unusual case of necrotizing fasciitis caused by $V$. vulnificus triggered by acupuncture needle insertion. The patient, who suffered from diabetes mellitus and nonalcoholic fatty liver disease and worked at a fish hatchery, denied any injury prior to acupuncture. This is the first ever reported case of $V$. vulnificus infection triggered by acupuncture needle insertion, clearly emphasizing the potential hazards of the prolonged survival of $V$. vulnificus on the skin. The potential infectious complications of acupuncture needle insertion are discussed.

\section{Introduction}

Vibrio vulnificus is a naturally occurring, free-living, inhabitant of estuarine and marine environments throughout the world, residing in high numbers in filter-feeding shellfish (oysters, clams, and mussels). ${ }^{1}$ This bacterium is considered one of the most dangerous waterborne bacterial pathogens and is responsible for the vast majority of seafood-related deaths worldwide. ${ }^{2,3}$ Human infections with $V$. vulnificus occur almost wherever the pathogen has been isolated, with reports mainly published from the USA, ${ }^{4}$ and Southeast Asia. ${ }^{5}$ However, disease cases have also been reported from Australia, Europe, South America, and the Mediterranean region. ${ }^{3,6}$

The two main types of infection caused by $V$. vulnificus are primary septicemia and wound infections. Primary septicemia which is characterized by fever, chills, and hypotension is the most serious and life-threatening feature of the disease and usually occurs following ingestion of raw or undercooked seafood. This type of infection comprises approximately 40$45 \%$ of all infections with $V$. vulnificus in the United States and Southeast Asia, where eating raw or undercooked seafood is a common practice. The case fatality rate of this type of infection could reach $\sim 50 \%{ }^{1}$ Wound infections occur when a patient suffers an injury before or during exposure to seawater or marine animals harboring the bacterium. Worldwide, the vast majority of human disease has been reported from USA and Southeast Asia, while reports from other parts of the world are largely sporadic and typically due to wound infection.

Immunocompromized patients are at the highest risk for developing severe complications. Chronic liver diseases, primarily liver cirrhosis, have been considered as the most influential factor predisposing patients for catastrophic complications. ${ }^{7}$ In addition, diabetes mellitus, malignancy, end-stage renal disease, chronic immunosuppressive therapy, and ironoverload associated conditions such as hemochromatosis and thalassemias are all associated with increased susceptibility to $V$. vulnificus infection.

Necrotizing fasciitis (NF) is a rapid and severe life-threatening infection and is considered a true medical emergency. NF associated with $V$. vulnificus infection can occur during both types of infections, primary septicemia and wound infection, and carries a frightening case fatality rate. ${ }^{8} \mathrm{NF}$ involves a rapidly progressive infection reaching the deep subcutaneous tissues and fascia causing considerable subcutaneous edema with purpura, ecchymoses, and hemorrhagic bullae, rapidly evolving into vascular occlusion or ischemia, tissue necrosis and gangrene.

In 1996 an outbreak of severe soft tissue infections caused by $V$. vulnificus erupted in Israel among fish farmers and fish consumers ${ }^{6}$ All the patients in Israel suffered from wound infection during handling live fresh fish cultivated in inland fish farms. Here we describe the first reported case of NF due to $V$. vulnificus triggered by acupuncture needle insertion, we discuss the potential infectious hazards of acupuncture and review the literature for similar cases.

\section{Case Report}

A 62 year-old-man suffering from obesity, diabetes mellitus, hyperlipidemia, and nonalcoholic liver cirrhosis was referred to the emergency department due to fever, chills, and progressive swelling of his left arm. The patient's spouse mentioned that 36 hours prior
Correspondence: Naiel Bisharat, Department of Medicine D, Emek Medical Center, Yitshak Rabin Boulevard 21, 18341 Afula, Israel.

Tel.: +972.4649.4520 - Fax: +972.4649.4518.

E-mail: bisharat_na@clalit.org.il

Key words: Acupuncture; necrotizing fasciitis; shellfish; Vibrio vulnificus.

Contributions: YK wrote the initial draft, SS gathered the relevant medical data and contributed his part to the initial draft, NB wrote the final draft.

Conflict of interest: the authors declare no potential conflict of interest.

Received for publication: 14 March 2015.

Revision received: 16 June 2015.

Accepted for publication: 25 June 2015.

This work is licensed under a Creative Commons Attribution NonCommercial 3.0 License (CC BYNC 3.0).

(C) Copyright Y. Kotton et al., 2015

Licensee PAGEPress, Italy

Infectious Disease Reports 2015; 7:5901

doi:10.4081/idr.2015.5901

to his admission he underwent acupuncture needle insertion along the anterior medial aspect and radial side of his left hand. The patient used to work as an assistant hatchery manager at an inland fish farm that cultivated fish for commercial use. The patient denied any injury prior to his illness. Thirty hours prior to his admission he complained of generalized weakness and fever. During the day prior to his admission his condition worsened rapidly with progressive swelling of his left arm. His physical examination upon hospital admission was remarkable for severe disorientation, his vital signs showed a blood pressure of $88 / 50 \mathrm{mmHg}, 124$ beats per minute, 38 breaths per minute, and $\mathrm{O}_{2}$ saturation at room air of $82 \%$. The physical findings were suggestive of necrotizing fasciitis with severe edema, ecchymoses, hemorrhagic bullae, and progressive tissue necrosis of two fingers (Figure 1). His laboratory studies were compatible with multi-organ failure with acute renal failure, rhabdomyolysis, disseminated intravascular coagulation, and elevated liver enzymes. The patient was treated with ceftriaxone, penicillin, and clindamycin pending culture results. The patient underwent surgical debridement with fasciotomy and amputation of 2 necrotic fingers within 24 hours of his arrival to the emergency department. Blood and wound cultures grew V. vulnificus. Antimicrobial susceptibility showed that the bacterium was susceptible to third-generation cephalosporins, fluoroquinolones, tetracyclines, aminoglycosides, 
and extended-spectrum penicillins. Clindamycin and penicillin were stopped and he was treated with ceftriaxone to complete two weeks of treatment. Biochemical and molecular characterization identified the bacterium as a biotype 3 strain, the main biotype responsible for human disease in Israel. In the first two weeks of his stay he received hemodialysis treatment for severe kidney failure with complete recovery of kidney function. During the $3^{\text {rd }}$ week he suffered from septic shock. The patient was treated with meropenem, vancomycin, vasopressors, and mechanical ventilation. Blood cultures grew Pseudomonas aeruginosa that was susceptible to meropenem. The patient required skin grafts due to extensive skin loss and underwent tracheostomy due to failed weaning from mechanical ventilation. He also suffered from critical illness polyneuropathy. His condition improved gradually and after 72 days of hospital stay he was referred to a rehabilitation facility where he stayed for another 2 months.

\section{Discussion}

The case described is the first ever reported case of $V$. vulnificus infection triggered by acupuncture needle insertion. The infection evolved despite the use of standard precautions before needle insertion including single use of disposable sterile needles and local disinfection with alcohol 70\%. At the time of presentation the first clinical impression was that his illness may have been caused by other more common bacterial pathogens capable of

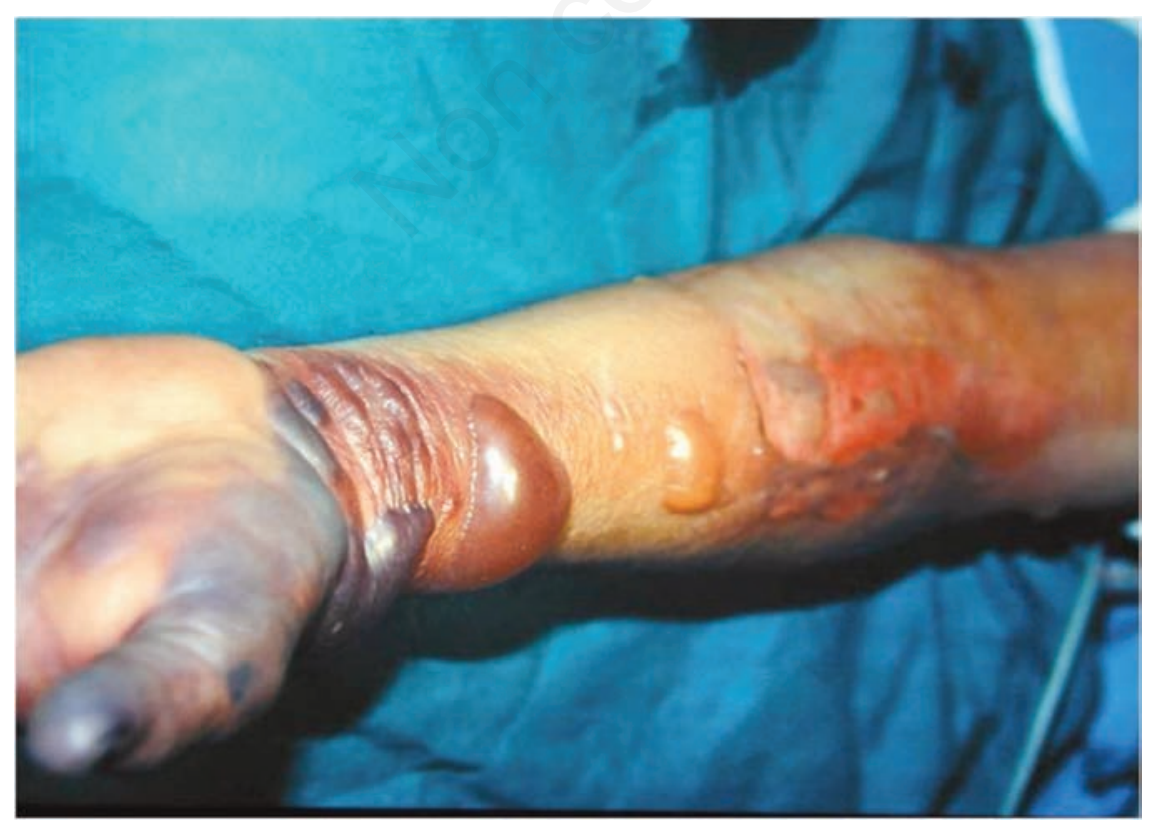

Figure 1. Left arm at hospital admission $36 \mathrm{~h}$ following acupuncture. causing NF such as Staphylococcus aureus or group-A $\beta$-Streptococcus. However, his exposure history of contact with brackish water accompanied by a short incubation time from acupuncture to symptom onset was highly suggestive of NF due to $V$. vulnificus. The patient denied any injury prior to acupuncture, this implies that $V$. vulnificus may have survived on the skin for at least one day before the acupuncture. The prolonged survival of $\mathrm{V}$. vulnificus on the skin has been previously demonstrated by other authors from Israel. ${ }^{9}$ To date, only three other cases of NF associated with acupuncture have been reported in the English literature, the causative pathogens in these cases were Pseudomonas aeruginosa, ${ }^{10} S$. aureus, ${ }^{11}$ Enterococcus faecalis and Gram-negative rods. ${ }^{12}$ Acupuncture associated infections due to vibrios have been reported only once, in non-English literature, the report described a Korean patient suffering from liver cirrhosis who developed non-01, non-0139 Vibrio cholera septicemia following acupuncture. ${ }^{13}$

Acupuncture has become extremely popular for pain management and other medical conditions. According to data released by the National Institutes of Health (NIH) in 2008 nearly 3.1 million American adults and 150,000 children used acupuncture in 2007. ${ }^{14}$

Acupuncture is usually considered to be a safe procedure with very few adverse effects, one review estimated that that serious adverse events occur in 0.55 per 10,000 acupuncture treatments. ${ }^{15}$ Infectious complications of acupuncture are nowadays extremely rare, previous observations indicated that the most common complications were viral hepatitis due to the use of reusable needles. ${ }^{16} \mathrm{~A}$ more recent systematic review identified only 239 reported cases of infections associated with acupuncture, ${ }^{17}$ all of which were bacterial without any reports of viral transmission. The vast majority (81\%) of these infections were associated with Mycobacterium (chelonae, abscessus, and haemophilum), while the rest included other bacteria such $S$. aureus, Escherichia coli, Klebsiella pneumoniae, P. aeruginosa, E. faecalis, and Listeria monocytogenes.

Necrotizing fasciitis caused by $V$. vulnificus is a fatal disease, especially among immunocompromized hosts and particularly patients with liver cirrhosis from any cause. The mainstay of treatment of patients with NF is timely antimicrobial therapy combined with surgical debridement and fasciotomy. The clinical manifestations of NF caused by $V$. vulnificus and other bacterial pathogens such as group-A $\beta$ Streptococcus, S. aureus, Aeromonas species, or $K$. pneumoniae are indistinguishable, making it difficult to differentiate between the different causative pathogens at the time of hospital admission. A group of authors from Taiwan addressed this issue by comparing the clinical characteristics, laboratory findings, and outcome of patients suffering from NF caused by $V$. vulnificus to NF caused by $S$. aureus, ${ }^{18}$ Aeromonas spp., ${ }^{19}$ and $K$. pneumoniae. ${ }^{20}$ They concluded that NF caused by $V$. vulnificus progresses more rapidly than NF caused by $S$. aureus or K. pneumoniae.

To date, the largest cohorts of NF caused by V. vulnificus were published by three different groups from Taiwan. ${ }^{18,19,21-28}$ These studies were sufficiently detailed and described different aspects of the disease such as clinical characteristics, ${ }^{18-20}$ laboratory data, ${ }^{21}$ timing of surgery, ${ }^{24}$ and antimicrobial therapy. ${ }^{25}$ Based on these cohorts the case fatality rate of NF following primary septicemia and wound infection averaged $55.7 \%$ and $28.9 \%$, respectively.

Most reports of human infections due to $V$. vulnificus have been reported from USA and Southeast Asia. However, the past 20 years had witnessed a significant increase in reports of human infections outside these regions mainly from Europe and the Middle East. This coincided with several ecological surveys showing that $V$. vulnificus thrives in water, shellfish and sediment along the Mediterranean coasts. ${ }^{29-33}$ It has been suggested by us, ${ }^{34}$ and others, ${ }^{35,36}$ that the emergence of $V$. vulnificus disease outside the traditional zones (i.e. USA and Southeast Asia) was mainly driven by climate change.

Antimicrobial susceptibility testing of $\mathrm{V} . \mathrm{vul}$ nificus strains from several areas showed that the bacterium is susceptible to third-generation cephalosporins, fluoroquinolones, tetracyclines, aminoglycosides, and extended-spectrum penicillins. ${ }^{37-40}$ To date, the vast majority of publications advocate the use of third-gen- 
eration cephalosporins plus tetracyclines as the initial antimicrobial therapy for patients with presumed NF caused by $V$. vulnificus. ${ }^{19,22,24,25,41,42}$ This combination therapy exhibited a synergistic effect against $V$. vulnificus ${ }^{43,44}$ and was associated with a better outcome among patients with $V$. vulnificus septicemia. ${ }^{41,45}$ Nonetheless, these recommendations are based on retrospective analysis of data combined with experimental evidence for the role of tetracyclines in eradicating $V$. vulnificus. ${ }^{46}$ Other treatment options that have been found to be also effective include fluoroquinolones plus minocycline (or analogue), penicillins plus aminoglycosides, ${ }^{24,25}$ and carbapenems. ${ }^{47}$ In our experience, ${ }^{6,48}$ and others in Israel, ${ }^{39}$ we did not find any advantage for a specific antimicrobial therapy among patients with $V$. vulnificus infection.

\section{Conclusions}

Patients with chronic liver disease are highly susceptible to invasive infections caused by V. vulnificus. These patients should be careful during exposure to brackish water and avoid any exposure to raw or undercooked marine animals. Acupuncture should always be carried out using clean care practices. The prolonged survival of $V$. vulnificus on the skin should prompt individuals to proper cleansing post exposure to brackish water or marine animals.

\section{References}

1. Jones MK, Oliver JD. Vibrio vulnificus: disease and pathogenesis. Infect Immun 2009;77:1723-33.

2. Feldhusen F. The role of seafood in bacterial foodborne diseases. Microbes Infect 2000;2:1651-60.

3. Oliver JD. Vibrio vulnificus. In: Thompson FL, Austin B, Swings JG, eds. The biology of vibrios. Washington, D.C.: ASM Press; 2006. pp. 349-366.

4. Gary Hlady W, Klontz KC. The epidemiology of Vibrio infections in Florida, 19811993. J Infect Dis 1996;173:1176-83.

5. Chuang YC, Yuan CY, Liu CY, et al. Vibrio vulnificus infection in Taiwan: report of 28 cases and review of clinical manifestations and treatment. Clin Infect Dis 1992;15:271-6.

6. Bisharat N, Agmon V, Finkelstein R, et al. Clinical, epidemiological, and microbiological features of Vibrio vulnificus biogroup 3 causing outbreaks of wound infection and bacteraemia in Israel. Israel Vibrio Study Group. Lancet 1999;354:1421-4.

7. Haq SM, Dayal HH. Chronic liver disease and consumption of raw oysters: a potentially lethal combination-a review of Vibrio vulnificus septicemia. Am J Gastroenterol 2005;100:1195-9.

8. Chen SC, Chan KS, Chao WN, et al. Clinical outcomes and prognostic factors for patients with Vibrio vulnificus infections requiring intensive care: a 10-yr retrospective study. Crit Care Med 2010;38: 1984-90.

9. Colodner R, Chazan B, Kopelowitz J, et al. Unusual portal of entry of Vibrio vulnificus: evidence of its prolonged survival on the skin. Clin Infect Dis 2002;34:714-5.

10. Saw A, Kwan MK, Sengupta S. Necrotising fasciitis: a life-threatening complication of acupuncture in a patient with diabetes mellitus. Singapore Med J 2004;45:180-2.

11. Hsieh RL, Huang CH, Uen WC. Necrotizing fasciitis after acupuncture in a patient with aplastic anemia. J Altern Complement Med 2011;17:871-4.

12. Macuha F Jr., Ahn A, Graham R. Necrotizing fasciitis associated with acupuncture: a case report. J Hosp Med 2010;5:565-6.

13. Lim TS, Ji A, Lee JH, Chang S, et al. Non01, non-0139, Vibrio cholerae septicemia after acupuncture. Ewha Med J 2013;36:S22-S4.

14. Barnes PM, Bloom B, Nahin RL. Complementary and alternative medicine use among adults and children: United States, 2007. Natl Health Stat Report 2008:1-23.

15. White A. A cumulative review of the range and incidence of significant adverse events associated with acupuncture. Acupunct Med 2004;22:122-33.

16. Lao L, Hamilton GR, Fu J, et al. Is acupuncture safe? A systematic review of case reports. Altern Ther Health Med 2003;9:7283.

17. Xu S, Wang L, Cooper E, et al. Adverse events of acupuncture: a systematic review of case reports. Evid Based Complement Alternat Med 2013;2013: 581203.

18. Tsai YH, Wen-Wei Hsu R, Huang KC, et al. Comparison of necrotizing fasciitis and sepsis caused by Vibrio vulnificus and Staphylococcus aureus. J Bone Joint Surg Am 2011;93:274-84.

19. Tsai YH, Hsu RW, Huang TJ, et al. Necrotizing soft-tissue infections and sepsis caused by Vibrio vulnificus compared with those caused by Aeromonas species. J Bone Joint Surg Am 2007;89:631-6.

20. Hsu JC, Shen SH, Yang TY, et al. Necrotizing fasciitis and sepsis caused by Vibrio vulnificus and Klebsiella pneumoniae in Diabetic patients. Biomed $\mathrm{J}$ 2015;38:136-42

21. Chao WN, Tsai SJ, Tsai CF, et al. The labo- ratory risk indicator for necrotizing fasciitis score for discernment of necrotizing fasciitis originated from Vibrio vulnificus infections. J Trauma Acute Care Surg 2012;73:1576-82.

22. Lee YC, Hor LI, Chiu HY, et al. Prognostic factor of mortality and its clinical implications in patients with necrotizing fasciitis caused by Vibrio vulnificus. Eur J Clin Microbiol Infect Dis 2014;33:1011-8.

23. Tsai YH, Hsu RW, Huang KC, et al. Laboratory indicators for early detection and surgical treatment of vibrio necrotizing fasciitis. Clin Orthop Relat Res 2010;468:2230-7.

24. Chao WN, Tsai CF, Chang HR, et al. Impact of timing of surgery on outcome of Vibrio vulnificus-related necrotizing fasciitis. Am J Surg 2013;206:32-9.

25. Chen SC, Lee YT, Tsai SJ, et al. Antibiotic therapy for necrotizing fasciitis caused by Vibrio vulnificus: retrospective analysis of an 8 year period. J Antimicrob Chemother 2012;67:488-93.

26. Kuo YL, Shieh SJ, Chiu HY, et al. Necrotizing fasciitis caused by Vibrio vulnificus: epidemiology, clinical findings, treatment and prevention. Eur J Clin Microbiol Infect Dis 2007;26:785-92.

27. Tsai YH, Hsu RW, Huang KC, et al. Systemic Vibrio infection presenting as necrotizing fasciitis and sepsis. A series of thirteen cases. J Bone Joint Surg Am 2004;86A:2497-502.

28. Tsai YH, Huang TJ, Hsu RW, et al. Necrotizing soft-tissue infections and primary sepsis caused by Vibrio vulnificus and Vibrio cholerae non-01. J Trauma 2009;66:899-905.

29. Arias CR, Olivares-Fuster 0, Goris J. High intragenomic heterogeneity of $16 \mathrm{~S}$ rRNA genes in a subset of Vibrio vulnificus strains from the western Mediterranean coast. Int Microbiol 2010;13:179-88.

30. Canigral I, Moreno Y, Alonso JL, et al. Detection of Vibrio vulnificus in seafood, seawater and wastewater samples from a Mediterranean coastal area. Microbiol Res 2010;165:657-64.

31. Cantet F, Hervio-Heath D, Caro A, et al. Quantification of Vibrio parahaemolyticus, Vibrio vulnificus and Vibrio cholerae in French Mediterranean coastal lagoons. Res Microbiol 2013;164:867-74.

32. Ghinsberg RC, Dror R, Nitzan Y. Isolation of Vibrio vulnificus from sea water and sand along the Dan region coast of the Mediterranean. Microbios 1999;97:7-17.

33. Normanno G, Parisi A, Addante N, et al. Vibrio parahaemolyticus, Vibrio vulnificus and microorganisms of fecal origin in mussels (Mytilus galloprovincialis) sold in the Puglia region (Italy). Int $\mathrm{J}$ Food Microbiol 2006;106:219-22. 
34. Paz S, Bisharat N, Paz E, et al. Climate change and the emergence of Vibrio vulnificus disease in Israel. Environ Res 2007;103:390-6.

35. Vezzulli L, Colwell RR, Pruzzo C. Ocean warming and spread of pathogenic vibrios in the aquatic environment. Microb Ecol 2013;65:817-25.

36. Baker-Austin C, Stockley L, Rangdale R, et al. Environmental occurrence and clinical impact of Vibrio vulnificus and Vibrio parahaemolyticus: a European perspective. Environ Microbiol Rep 2010;2:7-18.

37. Hsueh PR, Chang JC, Chang SC, et al. In vitro antimicrobial susceptibility of Vibrio vulnificus isolated in Taiwan. Eur J Clin Microbiol Infect Dis 1995;14:151-3.

38. Shaw KS, Rosenberg Goldstein RE, He X, et al. Antimicrobial susceptibility of Vibrio vulnificus and Vibrio parahaemolyticus recovered from recreational and commercial areas of Chesapeake Bay and Maryland Coastal Bays. PLoS One 2014;9:e89616.

39. Zaidenstein R, Sadik C, Lerner L, et al. Clinical characteristics and molecular subtyping of Vibrio vulnificus illnesses, Israel. Emerg Infect Dis 2008;14:1875-82.

40. Hsueh PR, Lin CY, Tang HJ, et al. Vibrio vulnificus in Taiwan. Emerg Infect Dis 2004;10:1363-8.

41. Liu JW, Lee IK, Tang HJ, et al. Prognostic factors and antibiotics in Vibrio vulnificus septicemia. Arch Intern Med 2006;166: 2117-23.

42. Morris JG Jr., Tenney J. Antibiotic therapy for Vibrio vulnificus infection. JAMA 1985;253:1121-2.

43. Chuang YC, Ko WC, Wang ST, et al. Minocycline and cefotaxime in the treatment of experimental murine Vibrio vulnificus infection. Antimicrob Agents Chemother 1998;42:1319-22.

44. Chuang YC, Liu JW, Ko WC, et al. In vitro synergism between cefotaxime and minocycline against Vibrio vulnificus.
Antimicrob Agents Chemother 1997;41: 2214-7.

45. Chiang SR, Chuang YC. Vibrio vulnificus infection: clinical manifestations, pathogenesis, and antimicrobial therapy. J Microbiol Immunol Infect 2003;36:81-8.

46. Bowdre JH, Hull JH, Cocchetto DM. Antibiotic efficacy against Vibrio vulnificus in the mouse: superiority of tetracycline. J Pharmacol Exp Ther 1983;225:5958.

47. Matsumoto K, Ohshige K, Fujita N, et al. Clinical features of Vibrio vulnificus infections in the coastal areas of the Ariake Sea, Japan. J Infect Chemother 2010;16: 272-9.

48. Bisharat N. Molecular and seroepidemiological studies to investigate the emergence of Vibrio vulnificus in Israel. Thesis Dissertation. Tel-Aviv University; 2009. 